\title{
EMDR for Childhood PTSD After Road Traffic Accidents: Attentional, Memory, and Attributional Processes
}

\author{
Tracy Ribchester \\ William Yule \\ Adam Duncan \\ King's College London, Institute of Psychiatry, London SE5 8AF, UK
}

\begin{abstract}
Eye movement desensitization and reprocessing (EMDR) was used with 11 children who developed posttraumatic stress disorder (PTSD) after road traffic accidents. All improved such that none met criteria for PTSD on standardized assessments after an average of only 2.4 sessions. Significant improvements in PTSD, anxiety, and depression were found both immediately after treatment and at follow-up. Attentional, memory, and attributional processes associated with PTSD were assessed and their relationship to therapeutic change examined. Treatment was associated with a significant trauma-specific reduction in attentional bias on the modified Stroop task, with results apparent both immediately after therapy and at follow-up.
\end{abstract}

Keywords: PTSD; child; EMDR; attention; memory; attribution

E ye movement desensitization and reprocessing (EMDR) therapy (Shapiro, 1989) has emerged as a rapid treatment for posttraumatic stress disorder (PTSD). The UK National Institute of Clinical Excellence (NICE, 2005) recently recommended EMDR for PTSD in adults, but noted that there is a smaller and less conclusive evidence base for children.

Early single case studies with children reported rapid treatment gains that were maintained after 6 months (Coco \& Sharpe, 1993; Pellicer, 1993). An open trial of five children showed gains in one to two sessions that were maintained or increased at 1-month follow-up (Greenwald, 1994). Two studies have reported using wait-list controls (EMDR versus no treatment). Puffer, Greenwald, and Elrod (1998) treated 20 children with traumatic memories using a single session of EMDR, with half receiving the treatment after a 1-month delay. Over half of the children moved from clinical to normal levels on The Impact of Event Scale, and all but three children showed at least partial symptom relief on some measures. However, there was nonrandom allocation to groups; the same individual carried out all assessments and interventions and was not fully trained in EMDR; and no formal diagnoses were obtained. Chemtob,
Nakashima, and Carlson (2002) reported on the treatment of 32 children with PTSD 1 year after exposure to Hurricane Iniki, using three sessions of EMDR. Random assignment, independent assessment, and both self-report and behavioral outcome measures were used. Posttreatment assessment revealed that, relative to wait-list controls, there were substantial reductions in the PTSD symptoms of the treatment group, and more modest reductions in anxiety and depression. Treatment gains were maintained at 6-month follow-up, and number of visits to the school nurse was significantly reduced. The children had all failed to respond to previous interventions.

Some recent studies have compared EMDR to cognitive behavioral therapy (CBT) for children. Jaberghaderi, Greenwald, Rubin, Dolatabadim, and Zand (2004) used CBT and EMDR treatment groups for Iranian girls who had posttraumatic stress symptoms following sexual abuse. The results indicated a significant and large treatment effect on self-reported PTSD symptoms, with a trend favoring EMDR over CBT-although there were only seven children in each group. Soberman, Greenwald, and Rule (2002) found that the addition of EMDR sessions to standard care for 14 boys with conduct problems resulted in significant and large reductions of memory-related 
distress and problem behaviors, as well as trends toward reduction of PTSD symptoms. With a somewhat larger sample size, De Roos, Greenwald, de Jongh, and Noorthoorn (2004) randomized children exposed to a fireworks factory explosion in the Netherlands to either CBT $(n=26)$ or $\operatorname{EMDR}(n=26)$. Significant reductions in PTSD were found in both groups - with significantly fewer sessions of EMDR needed (3.2 rather than 4) and larger effect sizes for EMDR on 6 of 8 measures at 3-month follow-up.

Ahmad, Larsson, and Sundelin-Wahlsten (2007) report a randomized control trial of 33 children who not only had received a diagnosis of PTSD at a clinic but also had evidence of having experienced some additional social stress. The treatment protocol demanded that all 17 children in the treatment group receive eight sessions of EMDR irrespective of progress. They scored significantly lower on a symptom scale compared with the wait-list controls. No follow-up was undertaken.

Hensel (2009) reported on the treatment outcome of 36 children aged 1 year 9 months to 18 years 1 month who had each been exposed to a single-incident traumatic event. The criterion measure was the PROPS (Greenwald \& Rubin, 1999), a parent report instrument. Thirty-two of the consecutive referrals received on average three treatment sessions. There was little change in PROPS scores during waiting for treatment followed by a significant drop in scores immediately after treatment. The gains maintained during a 6-month follow-up. Interestingly, gains were as good for children under 4 years of age.

Kemp, Drummond, and McDermott (2010) report a pilot wait-list control study comparing four sessions of EMDR $(N=13)$ with 6-week wait-list $(N=14)$ with children aged 6-12 years suffering persistent PTSD symptoms after a motor vehicle accident. They did not have formal diagnoses of PTSD. Significant improvements were reported on the Child Posttraumatic Stress Reaction Index as well as on the process measures of Subjective Units of Distress Scale (SUDS) and Validity of Cognition (VOC) Scale. Treatment gains were maintained at 12-month follow-up.

\section{Cognitive Variables}

One of the main criticisms of earlier studies of EMDR was that claims of efficacy were often based on subjective verbal report measures like the SUDS, which may be highly susceptible to demand characteristics and procedural artifacts (Foa \& Meadows, 1997; Keane, 1998). Critics additionally felt that researchers had failed to include measures eliciting the processes that had been claimed to underpin the purported mechanisms of change (Lohr, Tolin, \& Lilienfeld, 1998). Unlike CBT techniques, EMDR does not directly target cognitive variables, yet is claimed to bring about change at the cognitive level as a byproduct of adaptive processing at the neurophysiological level. Evidence of such effects in the course of EMDR treatment would therefore be valuable in clarifying the process and supporting findings of efficacy. McNally (1996) reported that measures of information processing bias, such as the emotional Stroop and dichotic listening tasks, appear associated with many anxiety disorders in adults. Studies have utilized these tasks to gain an objective, unbiased measure of reductions in attentional bias to emotionally laden information following effective treatment with CBT techniques (Lavy, van den Hout, \& Arntz, 1993; Mathews, Mogg, Kentish, \& Eysenck, 1995; Mattia, Heimberg, \& Hope, 1993). It seems likely that such measures would be valuable in clarifying both psychological process and outcome in EMDR research.

The current study evaluated EMDR by utilizing a multimodal assessment methodology, including selfreport questionnaires, structured diagnostic interviews, and computer tasks tapping into attentional and memory biases. These measures were administered before and after treatment with EMDR, in a series of children who had developed PTSD following involvement in road traffic accidents.

\section{Method}

\section{Participants}

Forty seven children (8-16 years of age), all of whom had experienced an Road Taffic Accident (RTA) between 6 months and 2 years previously were assessed. They were recruited from two sources-an Accident and Emergency department in the south of England and a Child and Adolescent Mental Health Service.

Nineteen (40.4\%) scored $\geq 17$ on the eight-item Child Impact of Event Scale (Yule, 1997; http://www. childrenandwar.org), and so were classified as probable PTSD cases. A PTSD diagnosis was subsequently confirmed by independent clinical assessment using the Anxiety Disorders Interview Schedule (ADIS). Eleven $(57.9 \%)$ of these cases agreed to participate in the current EMDR treatment study and were eligible to do so. Reasons for nonparticipation included refusal of treatment or participation (three), involvement in litigation (one), complex needs (one), living outside the service locality (one), and remission of symptoms before treatment (two). One child declined further 
treatment after one incomplete session, but did complete all assessments and was therefore included in the analyses on an intent-to-treat basis.

Participants did not differ significantly in age or gender from the PTSD cases who did not participate.

At the time of pretreatment assessment, the mean age of the group was 11 years and 10 months $(S D=2$ years), ranging from 8 years to 15 years and 4 months. As a group they were of average verbal ability and reading attainment, as assessed on the British Picture Vocabulary Scale and the Wechsler Objectives Reading Dimension. Six participants were male and five female. Three of the boys were siblings, as were two of the girls. Seven of the children had been involved in car accidents as passengers and four had been knocked over by a car. Three of these had been involved in more than one RTA, and four had experienced additional traumatic events. The current study focuses only on the work related to RTA trauma. See Table 1 for details.

\section{Procedure}

Assessments were carried out at four time points: an initial screening assessment occurred at varying times prior to the start of treatment (from 2 weeks to 310 days, mean $=127$ days); pretreatment assessment 1 week prior to the start; posttreatment assessment 1 week after termination; and follow-up assessment between 1 and 4 months after termination of treatment. The first author administered all initial screening tests, and computerized assessments for attention and memory. At pretreatment and posttreatment, all other assessments were conducted by a clinical psychologist who was not directly involved in the research project and who had no formal training in, or allegiance to, EMDR. She was not, however, blind to the clinical or treatment status of the child.

Approximately 1 week after pretreatment assessment, each child underwent weekly EMDR treatment consisting of sessions no longer than 60 minutes. For the current study, the focus was only on RTA trauma. To ensure treatment fidelity, the therapist (the first author) completed Level One and Child and Adolescent Specialist Level training in EMDR, and followed a stepby-step treatment protocol (Tinker \& Wilson, 1999). Sessions were videotaped with the permission of both the child and parent, and treatment fidelity was assessed on the basis of these tapes by an experienced EMDR trainer with no direct involvement in the study.

The following standardized questionnaires were used at all assessment points: The Child Revised Impact of Event Scale (CRIES; Dyregrov \& Yule, 1995, www.childrenandwar.org); The Revised Children's Manifest Anxiety Scale (RCMAS; Reynolds \& Richmond, 1978) is a well-established 37-item selfreport questionnaire with higher scores indicating greater levels of anxiety; The Depression SelfRating Scale (DSRS; Birleson, 1981) is an 18-item self-report questionnaire with good concurrent validity; Children's Attributional Style QuestionnaireRevised (CASQ-R; Kaslow \& Thompson, 1998) has 24 forced choice items with good psychometric properties. Previous research has indicated that adolescents who make more internal attributions are more likely to develop subsequent psychopathology (Joseph, Brewin, Yule, \& Williams, 1993); and the Children's Assumptive World's Scale (CAWS; Bishop, Dalgleish, \& Yule, 2000) has good internal reliability $(0.82)$ for positive assumptions and (0.70) for negative

TABLE 1. Subject Characteristics

\begin{tabular}{lcc}
\hline Subject Characteristics & Cases $=11$ & Ranges \\
\hline Gender ratio (m:f) & $6: 5$ & \\
Mean age at time of assessment (years) & $11.90(2.02)$ & $8.25-15.25$ \\
Mean age at time of injury (years) & $9.67(2.01)$ & $7.58-12.41$ \\
Time in months since accident & $26.73(17.03)$ & $2-51$ months \\
Mean WORD reading score & $93.27(14.93)$ & \\
Mean BPVS score & $90.36(12.15)$ & \\
Number involved in type of accident & \\
$\quad$ Passenger in car & 7 & \\
$\quad$ Pedestrian knocked over by car & 4 \\
\hline $\begin{array}{l}\text { Note. WORD }=\text { Wechsler Objective Reading Dimensions (basic reading, standard } \\
\text { score); BPVS = British Picture Vocabulary Scale. } \\
\quad \text { Three were involved in additional accidents. }\end{array}$
\end{tabular}


assumptions. Children who developed PTSD after RTAs had a tendency to report decreased endorsements of negative assumptions about the world. Two additional measures were taken during the treatment sessions themselves-SUDS (Wolpe, 1973) and VOC scale (Shapiro, 1995).

In addition, a computerized emotional Stroop task was used to assess attentional bias at all assessment points except initial screening. This involved presenting words in different colors on a computer screen, and the participant's task was to name the word not the color. One-hundred and twenty presentations were made, comprising 60 words repeated twice in different colors (two at random from red, blue, green, or yellow). The words were taken from Neshat-Doost, Moradi, Taghavi, Yule, and Dagleish (1999) and fell into five groups of 12 words: anxiety-related (e.g., petrified, kidnapped), depression-related (e.g., lonely, helpless), PTSD and RTA-related (e.g., casualty, police), positive emotional (grateful, funfair), and words classed as neutral (e.g., bird, robin).

Computerized memory tasks were also administered at screening and posttreatment. To test recall, a further 60 words falling into the same categories as noted earlier were presented. After repeating and thinking about each word as it was presented, and then completing a distraction task, participants wrote down as many words as they could recall in 5 minutes. A word was considered to be correctly recalled if it was in the same form as it had been presented in the test, e.g., noun, adjective, or verb, whether or not it was correctly spelled. To test recognition memory, the same 60 words were immediately presented again, mixed with 60 new "buffer" words falling into the same categories. At each presentation children indicated whether or not they had seen the word in the previous test. See Moradi, Taghavi, Neshat Doost, Yule, and Dagleish; and Moradi, Taghavi, Neshat Doost, Yule, and Dagleish $(1999,2000)$ for further details of the computerized tasks.

Finally, at pre- and posttreatment assessments, diagnostic clinical interviews (ADIS for DSM-IV: C and P: Albano \& Silverman, 1996) were carried out with both children and parents (PTSD, generalized anxiety disorder (GAD), and major depressive disorder (MDD) sections only). A diagnosis was assigned if criteria (based on endorsement of sufficient symptoms and impact on functioning rated four or more out of eight) were met in either the child or the parent interview. Separate symptom counts within each of the three domains of the PTSD section (intrusion, avoidance, and arousal) were calculated for each child.

\section{Results}

The time between assessments varied considerably between children. On the advice of a statistician, "change quotients" or "change per elapsed day" were therefore calculated for each measure. These were obtained by subtracting the scores at one time point from those at a previous time point and dividing by the number of days passed. This gave a measure of the rate of change per day from one assessment point to another-negative if scores had increased, indicating a worsening of symptoms, positive if scores had decreased. The mean rate of change between screening and pretreatment assessments (mean of 127 days - ranging from 14 to 310) thus indicated any spontaneous change in symptoms with no treatment. This baseline was then compared to the mean rate of change between pretreatment and posttreatment assessments (mean gap of 45 days, ranging from 14 to 78), indicating the immediate effects of the therapy. The mean rate of change between pretreatment and follow-up assessments (mean gap of 113 days, ranging from 64 to 162) indicated whether significant benefits relative to baseline were also apparent over a longer period. Treatment was terminated when scores on the eight-item Children's Revised Impact of Event Scale reduced to below clinical cutoff and when the child's SUDS ratings remained at 0 or 1 and VOC ratings remained at 7 . Based on these criteria, between one and four sessions were required to treat the PTSD symptoms related to the RTAs. See Figure 1 and Table 2 for results on the following standardized questionnaires.

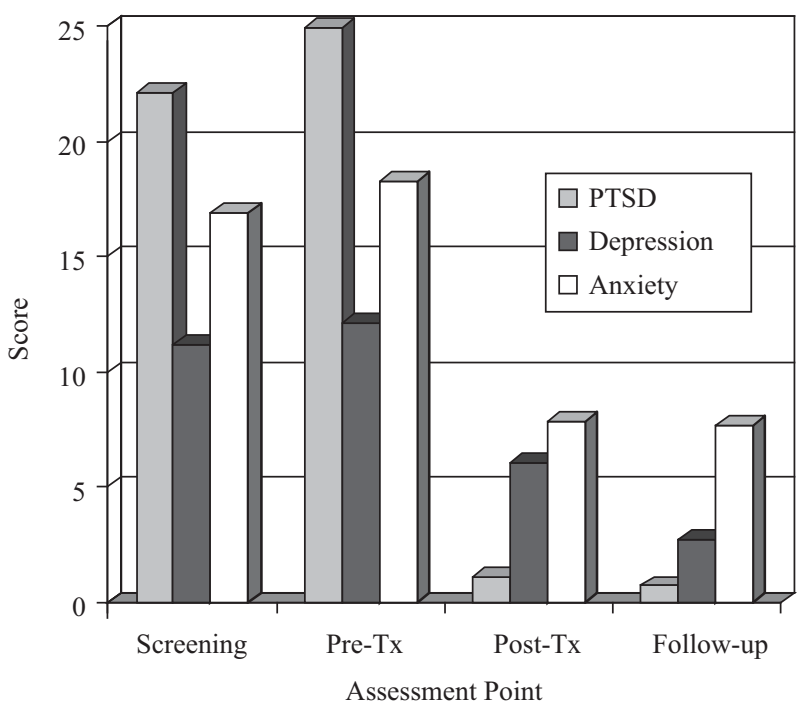

FIGURE 1. PTSD, depression, and anxiety questionnaire scores across assessment points. 
TABLE 2. Means (SD) and Change Quotients for Standardized Measures

\begin{tabular}{lccccc}
\hline & CRIES & RCMAS & DSRS & SUDS & VOC \\
\hline Screening (1) & $22.09(5.50)$ & $16.9(4.01)$ & $11.18(4.07)$ & N/A & N/A \\
Pretreatment (2) & $24.91(8.30)$ & $18.27(4.34)$ & $12.09(5.58)$ & $7.56(1.63)$ & $3.2(1.39)$ \\
Posttreatment (3) & $1.09(1.38)$ & $7.82(6.60)$ & $6.09(6.93)$ & $0.45(1.21)$ & $6.8(0.42)$ \\
Follow-up (4) & $0.73(1.10)$ & $7.27(6.63)$ & $2.82(2.99)$ & $0.45(0.93)$ & $7.00(0.00)$ \\
Change score & & & & \\
$\quad$ No treatment (1-2) & $-0.098(0.197)$ & $-0.003(0.037)$ & $0.042(0.127)$ & & \\
$\quad$ Short term (2-3) & $0.604(0.313)$ & $0.302(0.322)$ & $0.161(0.203)$ & & \\
$\quad$ Long term (2-4) & $0.236(0.137)$ & $0.100(0.090)$ & $0.085(0.051)$ & & \\
\hline
\end{tabular}

Note. CRIES = Child Revised Impact of Event Scale; RCMAS = The Revised Children's Manifest Anxiety Scale; DSRS = The Depression Self-Rating Scale; SUDS = Subjective Units of Distress; VOC = Validity of Cognition.

\section{Children's Impact of Event Scale}

The eight-item version of the child IES, assessing intrusion and avoidance symptoms only, was used in this analysis. Scores remained stable between screening and pretreatment, with a mean daily worsening of PTSD scores of -0.098 points. However, between pretreatment and posttreatment, there was a mean daily decrease in scores of 0.604 points. This represented highly significant short-term benefits relative to baseline, $t(10)=-6.796, p<.0001$. The rate of change between pretreatment and follow-up (0.236) also differed significantly from baseline, $t(10)=-3.952, p<.005$. Repeated measures analysis of variance (ANOVA) showed that the three rates differed significantly overall, $F(2,20)=29.025, p<.0001$. In regard to absolute child IES scores, between one and four sessions (mean $=2.36$ ) were required before scores were subclinical.

Taken together, the results suggest that treatment with EMDR led to a significant and rapid reduction in PTSD symptoms, which was apparent over both the immediate and longer term.

\section{RCMAS}

On this measure there was again very little change between screening and pretreatment (mean $=-0.003$ ). Between pretreatment and posttreatment, however, there was a mean daily improvement of 0.302 points, which was significantly different from the baseline, $t(10)=-3.191, p<.01$. The long-term rate of change $(0.100)$ was also significantly greater than baseline, $t(10)=-3.945, p<.01$, although significantly less than the short-term rate, $F(2,20)=8.017, p<.01$. The results therefore suggest that EMDR significantly reduced levels of general anxiety on average, both in the short and long term.

\section{DSRS}

No significant difference was found in the daily rates of change between the no-treatment time interval and either the short-term treatment interval, $t(10)=-1.283, p>.1$, or the long-term treatment interval, $t(10)=-0.784, p>0.1$. The repeated measures ANOVA confirmed these results, $F(2,20)=1.135, p>$ .1. Therefore it appeared that EMDR did not have a significant effect in reducing levels of depression.

These findings may have been unduly affected by the only child to show an increase in depression. He had terminated treatment after one incomplete session, but was included on an intent-to-treat basis. However, his large increases in depression appeared to coincide with the deteriorating mental health of his mother, who attempted suicide in the week before posttreatment assessment. For this reason, it was felt legitimate to exclude this case from the analysis of the depression data. There was now very little change between the screening and pretreatment time points (0.009). The rate of change between pre and posttreatment (an improvement of 0.202 points per day) was now significantly different from baseline, $t(9)=-2.931, p<.01$. The difference from baseline of the long-term rate of change $(0.085)$ remained marginally nonsignificant, $t(9)=-2.026, p=.07$. Overall, therefore, the effects of the EMDR treatment on depression appeared significant with one child's data excluded, at least in the short term.

\section{CASQ-R}

Two subscale scores were computed, representing attributional style in regard to positive and negative events separately. A further composite score, derived by subtracting the negative from the positive event scores, 
represented the overall level of depressive attribution (the lower the score, the more depressive the style). Daily change quotients were again calculated for each of these scores separately. Paired $t$-tests showed that there were no significant differences between time intervals on any of the daily rates of change scores. A repeated measures ANOVA was also nonsignificant. Treating these children with EMDR therefore did not appear to influence their attributional style.

\section{CAWS}

CAWS results also involved three scores, representing the extent of positive and negative assumptions and a composite score derived by subtracting the negative from the positive to give a "difference-betweensubscales score." The results mirrored those found for the CASQ-R. There were no statistically significant changes in the daily rates of change for the positive, negative, or composite measure over and above changes occurring in the absence of treatment. In other words, all scores on the CAWS remained stable over time and unaffected by treatment with EMDR.

\section{The Anxiety Disorders Interview for DSM-IV (ADIS for DSM-IV: C \& P)}

All children met DSM-IV criteria for PTSD before treatment, while no child met criteria following treatment). Paired $t$-tests comparing pre- and posttreatment symptom counts revealed significant changes in all symptom domains. On pretreatment scores, parents reported slightly fewer symptoms, $t(10)=$ 2.123, $p=.06$. See Table 3 for details.

In addition to PTSD, one child was diagnosed with MDD at pretreatment, but did not meet criteria at posttreatment assessment. Seven met criteria for GAD at pretreatment. Six no longer did so at posttreatment on the child version of the ADIS, although parents of three reported continued clinically significant symptoms in their child.

\section{SUDS and VOC Ratings}

Statistically significant improvements in both the SUDS scores, $F(2,20)=119.035, p>.0001$, and VOC ratings, $F(2,18)=35.568, p>.0001$, were found over the three time periods, effect sizes 4.33 and 2.57. See Table 2 for details.

\section{The Modified Stroop Color-Naming Test}

All Stroop measures were found to be stable between screening and pretreatment. Since screening
TABLE 3. PTSD Symptom Counts From Child and Parent ADIS Interviews

\begin{tabular}{|c|c|c|}
\hline & $\begin{array}{c}\text { Pretreatment } \\
\text { Symptom } \\
\text { Count }\end{array}$ & $\begin{array}{l}\text { Posttreatment } \\
\text { Symptom } \\
\text { Count }\end{array}$ \\
\hline \multicolumn{3}{|l|}{ Child } \\
\hline Total $^{\star \star \star}$ & $12.636(2.335)$ & $2.272(2.004)$ \\
\hline Reexperiencing ${ }^{\star \star \star}$ & $4.090(1.30)$ & $0.181(0.405)$ \\
\hline Avoidance ${ }^{\star \star \star}$ & $4.00(0.894)$ & $0.545(0.522)$ \\
\hline Arousal $^{\star \star \star}$ & $4.545(0.687)$ & $0.363(0.504)$ \\
\hline \multicolumn{3}{|l|}{ Parent } \\
\hline Total $^{\star \star \star}$ & $10.727(2.533)$ & $3.818(2.401)$ \\
\hline Reexperiencing ${ }^{\star \star \star}$ & $2.909(0.272)$ & $0.272(0.646)$ \\
\hline Avoidance ${ }^{\star \star \star}$ & $4.181(0.874)$ & $2.00(1.183)$ \\
\hline Arousal $^{\star \star}$ & $3.636(0.809)$ & $1.545(1.368)$ \\
\hline
\end{tabular}

data on the Stroop were only collected for seven of the 11 children due to computer problems, the scores at screening and pretreatment were averaged for those who had both scores. Thus for all eleven children there were three sets of data: pretreatment, posttreatment, and follow-up data. Response latencies and interference indexes for each word type were derived for each time point. See Table 4 for results.

At pretreatment none of the emotional word types had significantly longer response latencies than the neutral word types, although there was a trend for the children to take longer to color-name PTSD words, $t(10)=-1.928, p=.08$. At posttreatment, the PTSD words were named significantly faster than neutral words, $t(10)=2.946, p=.01$, while reaction times for all other word types remained unchanged. A similar trend for faster response latencies for PTSD words versus neutral words was found at follow-up but failed to reach significance, $t(9)=2.096, p=.06$. In absolute terms, PTSD response latencies were the only scores to change significantly over time, with response times becoming significantly quicker from pretreatment to posttreatment, $t(10)=2.680, p=$ .023 (see Figure 2). The level of interference that the children experienced from the trauma words was reduced significantly from pre- to posttreatment, $t(10)=2.946, p=.015$, unlike any other word type (see Figure 3). Taken together, these results indicate that EMDR had a specific effect on attentional bias, causing trauma-related words (and not words in other domains) to be named faster. 
TABLE 4. Means $(S D)$ for the Modified Stroop Task and Memory Recall Task

\begin{tabular}{lccc}
\hline & Pretreatment & Posttreatment & Follow-up \\
\hline Stroop task, words & & & \\
$\quad$ Neutral & $991.80(176.20)$ & $1017.85(256.94)$ & $1006.42(256.94)$ \\
Happy & $988.91(181.55)$ & $975.37(239.32)$ & $946.34(216.00)$ \\
Depressed & $1005.82(177.73)$ & $995.14(230.24)$ & $960.96(192.01)$ \\
Threat & $1010.38(181.16)$ & $991.44(213.06)$ & $1010.64(254.74)$ \\
PTSD & $1020.89(181.73)$ & $947.97(196.21)$ & $953.50(204.020)$ \\
Recall task, words & & & \\
Neutral & $3.36(1.96)$ & $3.09(1.81)$ & N/A \\
Happy & $2.36(1.36)$ & $1.91(1.22)$ & N/A \\
Depressed & $1.81(1.25)$ & $2.27(1.34)$ & N/A \\
Threat & $0.91(1.14)$ & $1.18(1.07)$ & N/A \\
PTSD & $4.00(1.34)$ & $2.82(1.07)$ & N/A \\
False recall & $1.00(1.41)$ & $2.09(2.11)$ & N/A \\
Total recall & $12.55(3.41)$ & $11.18(3.54)$ & N/A \\
\hline
\end{tabular}

Note. $\mathrm{PTSD}=$ posttraumatic stress disorder; $\mathrm{N} / \mathrm{A}=$ not applicable.

\section{The Recall Memory Test}

The total number of PTSD words recalled was significantly reduced following treatment, $t(10)=2.951$, $p=.014$, while the recall of all other word types was unchanged. Children recalled significantly fewer threat words than neutral words both before, $t(10)=3.688, p=.005$, and after treatment, $t(10)=4.605, p=.001$. See Table 4 for means.

\section{The Recognition Memory Test}

For each word type, both $\mathrm{d}^{\prime}$ (sensitivity) and $\beta$ (response bias) were calculated for each child at pre- and posttreatment assessments. However, no comparisons proved statistically significant, either between word types or between pre- and posttreatment assessments.

\section{Discussion}

Analyses of a case series showed that the PTSD symptoms of all 11 children remitted to subclinical levels after one to four sessions of EMDR - an average of just 2.4 sessions per child. Significant improvements over the longer term were also found. Standardized clinical interviews with parent and child gave further weight to the clinical efficacy of EMDR, showing highly significant clinical improvement in all three domains of PTSD as identified by either parent or child. Significant reductions in anxiety and depression symptoms were observed, even though the EMDR addressed traumatic memories.

A secondary question addressed by the current research was whether, when EMDR successfully treats the clinical signs of PTSD, does it then also affect the cognitive processing biases hypothesized to be involved in the disorder (Brewin, Dalgleish, \& Joseph, 1996; Ehlers \& Clark, 2000; Foa \& Kozak, 1986)? Treatment was associated with a significant trauma-specific reduction in attentional bias on the modified Stroop task. Response latencies for trauma words became significantly quicker and the level of interference produced by trauma words was significantly reduced, while latencies and levels of interference produced by all other word types remained unaffected. These results were apparent both immediately after therapy and at follow-up. Whether change at this attentional level mediated or only correlated with the effects of the therapy on PTSD levels cannot be answered in this study.

Although there was no evidence of a selective memory bias toward trauma information at pretreatment, significantly fewer PTSD words (but not any other category of words) were recalled after treatment than before. The results suggest that an explicit memory bias toward trauma material may not be involved in the maintenance of a PTSD diagnosis in children, although there may be some connection between 


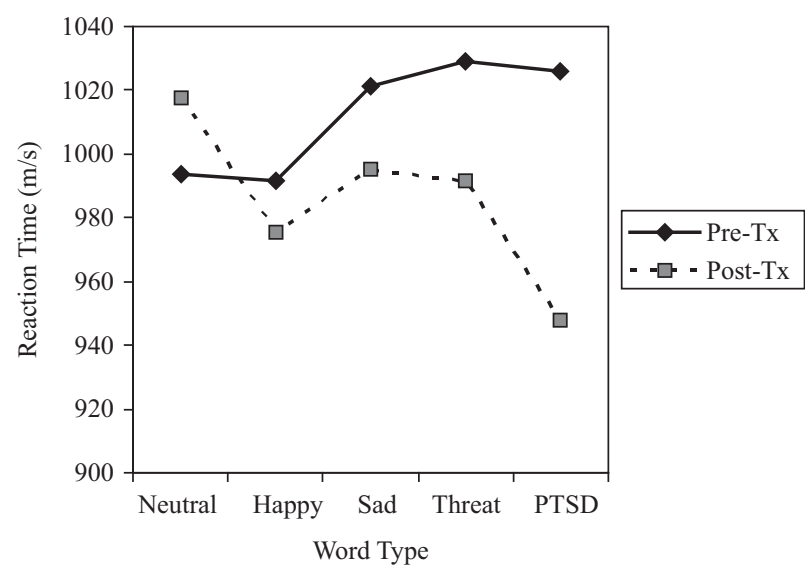

FIGURE 2. Latency scores by word type on the emotional Stroop task.

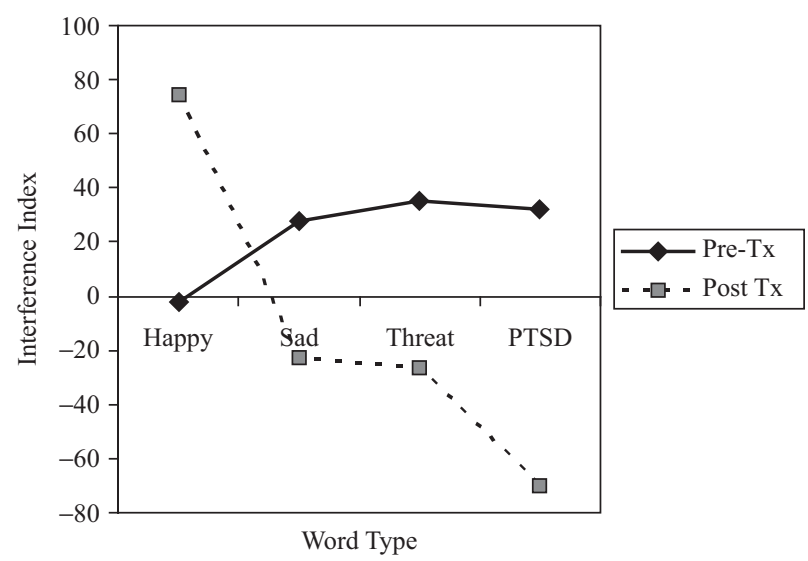

FIGURE 3. Interference scores by word type on the emotional Stroop task.

absolute level of recall and the severity of symptoms. Future research would need to assess the stability of this memory processing bias over a no-treatment time period, to provide a longer baseline measure against which to assess the impact of EMDR.

Cognitive models also suggest that attributions and appraisals made following a trauma can impede emotional processing if they are out of line with preexisting models of the world. In the current study, however, neither of the measures used to tap attributions and appraisals changed following EMDR. Examination of the negative and positive cognitions that children provided during the assessment phase of treatment, however, suggested these dimensions could still provide useful information. The majority of negative beliefs elicited appeared to focus on the child's appraisal of threat ("I am in danger"). There was also evidence of locus of control being an important factor in their appraisals ("I am not in control"), and with some other children, themes of guilt were an issue ("I am to blame").
In reviews of previous studies (Foa \& Meadows, 1997; Keane, 1998; Lohr et al., 1998), it has been recommended to use measures other than purely subjective outcome measures like the SUDS. It has also been recommended that studies include measures to evaluate the purported mechanisms of change in EMDR. Consequently, the present study used standardized clinical measures and ratings by parents, in addition to subjective SUDS and VOC ratings. Assessment was not limited to self-report but included computerized assessments of information processing biases such as the modified Stroop test, and diagnostic interviews and questionnaire assessment.

The first author administered all treatments, and the possibility that treatment gains were influenced by personal style and competence cannot therefore be ruled out. However, EMDR is a relatively highly standardized treatment; the clinician had formal training to child and adolescent specialist levels and followed a manualized treatment protocol. The sessions were also videotaped and evaluated by experienced child trainers. The case study design and absence of a waitlist control in the current study cannot rule out the possibility that EMDR had no effect over and above nonspecific treatment factors. However, it did allow for a comparison between the degree of change between screening and pretreatment assessment on each measure, and of degree of change following the start of treatment and at follow-up. Indeed, this procedure indicated the presence of treatment gains in 10 replicated case studies, over and above change occurring without intervention.

The data thus provide further evidence that EMDR is an effective and rapid treatment of single-incident PTSD in children.

\section{References}

Ahmad, A., Larsson, B., \& Sundelin-Wahlsten, V. (2007). EMDR treatment for children with PTSD: Results of a randomized controlled trial. Nordic Journal of Psychiatry, 61, 349-354.

Albano, A. M., \& Silverman, W. K. (1996). Anxiety Disorders Interview Schedule for DSM-IV: Clinical manual. San Antonio, TX: Psychological Corporation.

Birleson, P. (1981). The validity of depressive disorder in childhood and the development of self-rating scale. Journal of Child Psychology and Psychiatry, 22, 73-88.

Bishop, S. J., Dalgleish, T., \& Yule, W. (2000). Development of a children's measure of assumptive worlds. Unpublished doctoral dissertation, University of London.

Brewin, C. R., Dalgleish, T., \& Joseph, S. (1996). A dual representation theory of post-traumatic stress disorder. Psychological Review, 103, 670-686. 
Chemtob, C. M., Nakashima, J., \& Carlson, J. G. (2002). Brief treatment for elementary school children with disaster-related posttraumatic stress disorder: A field study. Journal of Clinical Psychology, 58, 99-112.

Coco, N., \& Sharpe, L. (1993). An auditory variant of eye movements desensitization in a case of childhood posttraumatic stress disorder. Journal of Behaviour Therapy and Experimental Psychiatry, 24, 373-377.

De Roos, C., Greenwald, R., de Jongh, A., \& Noorthoorn, E. O. (2004, November). EMDR versus CBT for disaster exposed children: A controlled study. Poster presented at the International Society for Traumatic Stress Studies, New Orleans, LA.

Dyregrov, A., \& Yule, W. (1995, May). Screening measures: The development of the UNICEFF screening battery. Paper presented at the 4th European Conference on Traumatic Stress, Paris.

Ehlers, A., \& Clark, D. M. (2000). A cognitive model of posttraumatic stress disorder. Behaviour Research and Therapy, 38, 319-345.

Foa, E. B., \& Kozak, M. J. (1986). Emotional processing of fear: Exposure to corrective information. Psychological Bulletin, 99, 20-35.

Foa, E. B., \& Meadows, E. A. (1997). Psychosocial treatments for posttraumatic stress disorder: A critical review. Annual Review of Psychology, 48, 449-480.

Greenwald, R. (1994). Applying eye movement desensitisation and reprocessing (EMDR) to the treatment of traumatised children: Five case studies. Anxiety Disorders Practice Journal, 1, 83-97.

Greenwald, R., \& Rubin, A. (1999). Brief assessment of children's post-traumatic symptoms: Development and preliminary validation of parent and child scales. Research on Social Work Practice, 9, 61-75.

Hensel, T. (2009). EMDR with children and adolescents after single-incident trauma. Journal of EMDR Practice and Research, 3, 2-9.

Jaberghaderi, N., Greenwald, R., Rubin, A., Dolatabadim, S., $\&$ Zand, S. O. (2004). A comparison of CBT and EMDR for sexually abused Iranian girls. Clinical Psychology and Psychotherapy, 11, 358-368.

Joseph, S., Brewin, C. R., Yule, W., \& Williams, R. (1993). Causal attributions and psychiatric symptoms in adolescent survivors of disaster. Journal of Child Psychology and Psychiatry, 34, 247-253.

Kaslow,N., \&Thompson,M.(1998).Children'sAttributional Style Questionnaire-Revised: Psychometric examination. Psychological Assessment, 10, 166-170.

Keane, T. M. (1998). Psychological and behavioural treatment of post-traumatic stress disorder. In P. Nathan \& J. Gorman (Eds.), Guide to treatments that work (pp. 398-407). Oxford: Oxford University Press.

Kemp, M., Drummond, P., \& McDermott, B. (2010). A wait-list controlled pilot study of eye movement desensitization and reprocessing (EMDR) for children with post-traumatic stress disorder (PTSD) symptoms from motor vehicle accidents. Clinical Child Psychology and Psychiatry, 15, 5-25.

Lavy, E. H., van den Hout, M., \& Arntz, A. (1993). Attentional bias and spider phobia: Conceptual and clinical issues. Behaviour Research and Therapy, 31, $17-24$.

Lohr, J. M., Tolin, D. F., \& Lilienfeld, S. O. (1998). Efficacy of eye movement and desensitisation and reprocessing: Implications for behaviour therapy. Behaviour Therapy, 29, 123-156.

Mathews, A., Mogg, K., Kentish, J., \& Eysenck, M. (1995). Effects of psychological treatment on cognitive bias in generalised anxiety disorder. Behaviour Research and Therapy, 33, 293-303.

Mattia, J. I., Heimberg, R. G., \& Hope, D. A. (1993). The revised Stroop colour-naming task in social phobics. Behaviour Research and Therapy, 31, 305-313.

McNally, R. J. (1996). Cognitive bias in the anxiety disorders. Nebraska Symposium on Motivation, 43, 211-250.

Moradi, A. R., Taghavi, M. R., Neshat Doost, H. T., Yule, W., \& Dagleish, T. (1999). Performance of children and adolescents with PTSD on Stroop colour naming task. Psychological Medicine, 29, 415-419.

Moradi, A. R., Taghavi, M. R., Neshat Doost, H. T., Yule, W., \& Dagleish, T. (2000). Memory bias for emotional information in children and adolescents with posttraumatic stress disorder: A preliminary study. Journal of Anxiety Disorders, 14, 521-534.

Neshat-Doost, H. T., Moradi, A. R., Taghavi, S. M. R., Yule, W., \& Dagleish, T. (1999). The developmental of a corpus of emotional words used by children and adolescents. Personality and Individual Differences, 27, 433-451.

National Institute for Clinical Excellence. (2005). Post traumatic stress disorder: The management of PTSD in adults and children in primary and secondary care (Clinical Guideline 26). London: Gaskell and the British Psychological Society.

Pellicer, X. (1993). Eye movement desensitization treatment of a child's nightmares: A case report. Journal of Behaviour Therapy and Experimental Psychiatry, 24, 73-75.

Puffer, M. K., Greenwald, R., \& Elrod, D. E. (1998). A single session EMDR study with twenty traumatized children and adolescents. Traumatology, 3. Retrieved from http://www.fsu.edu/ trauma/v3i2art6.html

Reynolds, C. R., \& Richmond, B. O. (1978). What I think and feel: A revised measure of children's manifest anxiety. Journal of Abnormal Child Psychology, 6, 271-280.

Shapiro, F. (1989). Eye movement desensitization: A new treatment for post-traumatic stress disorder. Journal of Behavioral Therapy and Experimental Psychiatry, 20, 211-217.

Shapiro, F. (1995). Eye movement desensitization and reprocessing: Basic principles, protocols and procedures. New York: Guilford Press. 
Soberman, G. B., Greenwald, R., \& Rule, D. L. (2002). A controlled study of eye movement desensitization and reprocessing (EMDR) for boys with conduct problems. Journal of Aggression, Maltreatment and Trauma, 6, 217-236.

Tinker, R. H., \& Wilson, S. A. (1999). Through the eyes of a child: EMDR with children. New York: W. W. Norton.

Wolpe, J. (1973). The practice of behaviour therapy. New York: Pergamon Press.

Yule, W. (1997). Anxiety, depression and post-traumatic stress in childhood. In I. Sclare (Ed.), Child psychology portfolio. Windsor: NFER-Nelson.
Acknowledgments. The authors would like to thank Mr. G. D. Bryant FRCS, FFAEM, Consultant at the Royal Sussex Hospital A \& E Department, without whose help the study would not have been possible. We would also like to thank the children and parents who participated in the study.

Correspondence regarding this article should be directed to William Yule, Department of Psychology (PO78), Institute of Psychiatry, De Crespigny Park, London, SE5 8AF, UK. E-mail: william.yule@kcl.ac.uk 